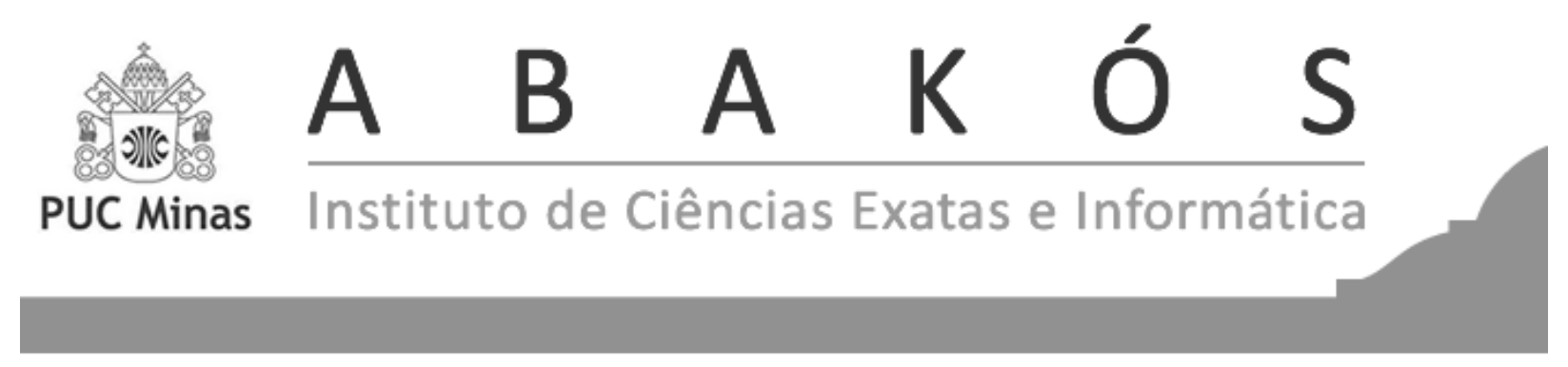

cc) (†)

\title{
Ensino de Astronomia: Tendências das Teses e Dissertações Publicadas Entre 2013 e 2019*
}

Astronomy Education: Trends of Theses and Dissertations Published Between 2013 and 2019

\author{
Cleonir Coelho Simões ${ }^{1}$ \\ Marcos Rincon Voelzke ${ }^{2}$ \\ Wagner Barbosa de Lima Palanch ${ }^{3}$
}

\begin{abstract}
Resumo
$\mathrm{O}$ presente artigo consiste em um mapeamento realizado nos principais Bancos de Teses e Dissertações entre os anos de 2013 e 2019, com o objetivo de verificar a presença de publicações sobre o Ensino de Astronomia. Busca-se responder à seguinte questão de pesquisa: Quais são as tendências metodológicas e temáticas evidenciadas nas Teses e Dissertações publicadas no período compreendido entre janeiro de 2013 e novembro de 2019? Trata-se de uma pesquisa qualitativa e do tipo Estado da Arte. Obtiveram-se 289 publicações, das quais, 27 correspondem a Teses de Doutorado e 262 a Dissertações de Mestrado. Destacamse o crescimento do número de publicações no período analisado, o Ensino Médio como o nível de ensino contemplado pelo maior número de publicações e Conteúdo-Método, Formação de Conceitos e Recursos Didáticos como os focos temáticos mais frequentes nas Teses e Dissertações analisadas.
\end{abstract}

Palavras-chave: Ensino de astronomia. Ensino de ciências e matemática. Estado da arte.

\footnotetext{
*Submetido em 05/02/2019 - aceito em 24/02/2021

${ }^{1}$ Universidade Cruzeiro do Sul e Instituto Federal de Minas Gerais, Brasil- cleonir.simoes@ifmg.edu.br

${ }^{2}$ Universidade Cruzeiro do Sul, Brasil- mrvoelzke@ hotmail.com

${ }^{3}$ Universidade Cruzeiro do Sul, Brasil- wagnerpalanch@gmail.com
} 


\begin{abstract}
This article consists of a mapping carried out in the main repositories for Theses and Dissertations between the years 2013 and 2019, intending to verify the presence of publications on Astronomy education. The aim is to answer the following research question: What are the methodological and thematic trends evidenced in these theses and dissertations? This is a qualitative and state of the art type of research. There were 289 publications, of which 27 corresponded to Doctoral Theses and 262 to Master's Dissertations. It is worth mentioning the growth in the number of publications in the period under review, the majority of them focusing on High School level, and Content-Method, Formation of Concepts and Didactic Resources as the most frequent thematic focuses in the Theses and Dissertations analyzed.
\end{abstract}

Keywords: Astronomy education. Science and mathematics education. State of the art. 


\section{INTRODUÇÃO}

Astronomia é "o estudo dos fenômenos celestes" (HOVATH, 2008, p. 13). Para esse autor, o interesse por essa ciência pode ter surgido da curiosidade dos seres humanos pelos céus (HORVATH, 2008). De acordo com Ivanissevich, Wuensche e Rocha (2010), a observação do céu em busca de simbolismos é uma prática que ocorre a milênios e, para Horvath (2008) e Afonso e Nadal (2013), o abandono do estilo nômade de vida e o cultivo da terra pelo homem pré-histórico podem ter contribuído para o desenvolvimento de um conhecimento astronômico rudimentar, fundamentado nas relações existentes entre os movimentos descritos pelos astros, sobretudo pelo Sol e pela Lua, e as variações cíclicas observadas na natureza.

O aperfeiçoamento das técnicas de observação do céu e o desenvolvimento de instrumentos astronômicos fizeram com que a Astronomia deixasse de ser uma ciência específica e bem consolidada, e se tornasse muito abrangente (PEIXOTO, 2018), chegando a despertar o interesse e a curiosidade de muitos, ao ponto de se destacar como uma ciência multidisciplinar (PEIXOTO; RAMOS, 2011), fascinando várias gerações e contribuindo para o desenvolvimento científico e tecnológico (MACÊDO, 2014; MORAES et al., 2016). De acordo com Macêdo (2014) e Moraes et al. (2016), muitos dos equipamentos utilizados na atualidade foram desenvolvidos a partir de descobertas astronômicas.

Em ambiente escolar, a Astronomia assume caráter motivador, tanto para alunos quanto para professores, e caráter multidisciplinar, uma vez que pode oferecer alternativas de abordagem para temas diversos e promover discussões técnicas, históricas e filosóficas com o público de educandos (GAMA; HENRIQUE, 2010). Além disso, caracteriza-se por ser uma ciência popular, ao alcance de professores e de alunos, uma vez que seu laboratório de pesquisa, o céu, se encontra à disposição de todos (LANGHI, 2009).

Por essas e outras razões que conteúdos de Astronomia estão presentes na estrutura curricular do Ensino Fundamental e do Ensino Médio (BRASIL, 2018), sendo ofertados, também, em alguns cursos de graduação como o Bacharelado e a Licenciatura em Física (BRETONES, 1999; PINTO et al., 2009, SAVALL; DUTRA, 2014). Entretanto, os conteúdos de Astronomia costumam ser apresentados de forma fragmentada e, não raro, como disciplina optativa (BRASIL, 2010; SAVALL; DUTRA, 2014).

Ainda no Ensino Superior, Gebara (2009) ressalta, em sua Tese de Doutorado, a presença da Astronomia no curso de Geografia, sobretudo na disciplina de Cartografia. Ziccardi (2009) destaca o fato de que a Astronomia se fazia presente no conteúdo programático da disciplina Mecânica Celeste, ofertada nos cursos de Matemática, logo após a sua criação no Brasil, mas acabou sendo retirada devido às reformulações sofridas por esses cursos no decorrer dos anos. 
Além de estar presente na Educação Básica e no Ensino Superior, a Astronomia também pode ser encontrada fora da sala de aula como em livros, revistas, programas de rádio e televisão, clubes de Astronomia, grupos de astrônomos amadores e na internet (FRAKNOI, 1999). Esses e outros aspectos têm despertado o interesse de pesquisadores que buscam compreender melhor a importância do Ensino da Astronomia.

Nos diversos trabalhos existentes sobre o tema, os pesquisadores buscam, por exemplo, analisar o conteúdo de Astronomia presente nos livros didáticos do Ensino Fundamental (COELHO; BULEGON, 2013) e do Ensino Médio (SIMÕES, 2009; DARROZ et al., 2017), os erros conceituais presentes nesses livros didáticos (LANGHI; NARDI, 2007; MORAIS et al., 2012), as concepções astronômicas apresentadas por professores (GONZAGA; VOELZKE, 2011) e alunos (BARBOSA; VOELZKE, 2016; MORAES et al., 2016), a formação continuada de professores (LANGHI, 2009; TEIXEIRA, 2013; MACEDO, 2014; BATISTA, 2016); e o uso das tecnologias digitais no ensino da Astronomia (MACÊDO et al., 2014; MACÊDO; VOELZKE, 2014; SIMÕES; VOELZKE, 2020).

Há, também, pesquisadores como Percy (2006), Gama e Henrique (2010), Soler e Leite (2012) e Langhi e Nardi (2014), que justificam o ensino de conteúdos de Astronomia em sala de aula. Dentre as justificativas apresentadas, destacam-se a relevância sócio-histórico-cultural dessa ciência, o seu caráter interdisciplinar, a sua capacidade de motivar o estudo de outras ciências e de ampliar a visão de mundo e, por fim, o potencial de promover a interação dos estudantes com a comunidade profissional e com espaços não formais de ensino.

O presente trabalho apresenta os resultados do mapeamento de pesquisas que abordam o Ensino de Astronomia e tem como principal objetivo verificar a presença de Teses e Dissertações relacionadas a esse tema, tomando-se como referência as áreas de Educação e de Ensino e tendo como fontes, os principais repositórios nacionais.

Buscou-se responder à seguinte questão: Quais são as tendências metodológicas e temáticas evidenciadas nas Teses e Dissertações recentemente publicadas, tomando-se como referência o tema Ensino de Astronomia? Para atingir o objetivo proposto e responder à questão que norteia esta pesquisa, realizou-se um levantamento bibliográfico das Teses e Dissertações produzidas no intervalo de tempo compreendido entre janeiro de 2013 e novembro de 2019, e que têm como tema o Ensino de Astronomia.

Trata-se de uma pesquisa de natureza qualitativa e constitui o que Ferreira (2002) denomina de Estado da Arte ou Estado do Conhecimento: 
Definidas como de caráter bibliográfico, elas parecem trazer em comum o desafio de mapear e de discutir certa produção acadêmica em diferentes campos do conhecimento, tentando responder que aspectos e dimensões vêm sendo destacados e privilegiados em diferentes épocas e lugares, de que formas e em que condições têm sido produzidas certas dissertações de mestrado, teses de doutorado, publicações em periódicos e comunicações em anais de congressos e de seminários. (FERREIRA, 2002, p.258)

Ainda, segundo Prigol (2013), esse tipo de estudo possui caráter bibliográfico e visa levantar dados sobre o conhecimento produzido sobre um tema, possibilitando uma visão do que outros pesquisadores publicaram.

Romanowski e Ens (2006) justificam a importância das pesquisas do tipo Estado da Arte para o avanço da ciência. Segundo essas autoras:

\footnotetext{
Esses estudos são justificados por possibilitarem uma visão geral do que vem sendo produzido na área e uma ordenação que permite aos interessados perceberem a evolução das pesquisas na área, bem como suas características e foco, além de identificar as lacunas ainda existentes. (ROMANOWSKI; ENS 2006, p. 39)
}

Por fim, Bretones e Megid Neto (2005) também ressaltam a importância desse tipo de estudo, pois contribuem para a divulgação da produção acadêmica, promovendo a socialização dos conhecimentos e apontando contribuições para o ensino.

Assim sendo, serão apresentadas nas seções que seguem a descrição detalhada dos procedimentos realizados durante este mapeamento e análise dos dados obtidos, fornecendo-se uma visão geral das Teses e Dissertações analisadas e, finalizando o presente trabalho, apresentam-se as considerações finais.

\section{PERCURSO METODOLÓGICO}

O primeiro passo do presente trabalho foi o levantamento das Teses e Dissertações que constituem o seu corpus. Esse levantamento foi realizado por meio do Banco de Teses e Dissertações da CAPES, da Biblioteca Digital Brasileira de Teses e Dissertações (BDTD), da Biblioteca Digital de Teses e Dissertações da Universidade de São Paulo (USP) e do Banco de Teses e Dissertações sobre Educação em Astronomia (BTDEA) da Universidade Federal de São Carlos (UFSCar), em que se utilizou como termo de busca, a palavra Astronomia.

O refinamento foi realizado inserindo-se os filtros "Educação", "Ensino" e "Ensino de Ciências e Matemática" no campo Área do Conhecimento e da escolha do período considerado para a pesquisa, que ficou compreendido entre janeiro de 2013 e novembro de 2019. Ressalta-se 
o fato de que, no refinamento, não se utilizou o filtro "Ensino de Astronomia", pois o mesmo não se encontrava presente no Banco de Teses e Dissertações da CAPES. Essa postura possibilitou um levantamento padronizado, isto é, realizado nas mesmas condições em todos os bancos envolvidos.

Justifica-se a escolha desse período pelo fato de todos os resultados nele contidos já se encontrarem catalogados na Plataforma Sucupira, o que simplificou consideravelmente o acesso aos dados. Além disso, constatou-se a existência de levantamentos desta natureza realizados em períodos anteriores a 2013 como, por exemplo, Siemsen e Lorenzetti (2017), Ferreira e Voelzke (2013), Bretones e Megid Neto (2005) e Megid Neto (1999).

O refinamento das buscas e o cruzamento dos resultados obtidos nos bancos analisados possibilitaram a contabilização de 289 publicações. Ressalta-se que, no caso do BTDEA, tal refinamento não foi possível e nem necessário, graças às suas características e excelente organização dos dados.

Iniciou-se, em seguida, a catalogação dos dados obtidos, procedimento que foi realizado utilizando-se ferramentas como o recurso "print screen", que cria uma imagem das informações exibidas na tela do computador e que pode ser manipulada por meio de um editor gráfico como, por exemplo, MSPaint, que é parte integrante dos sistemas operacionais da Microsoft ${ }^{\mathrm{TM}}$ (PEREIRA; MACHADO, 2008) ou o Kolourpaint, que integra o pacote de software livre do $K$ Desktop Environment (KDE) (DANG et al., 2011) e que, no caso, foi a ferramenta escolhida para esse trabalho. Esse procedimento foi fundamental para que se preservassem os resultados obtidos na data de realização final do levantamento, evitando-se que alterações posteriores nos bancos de dados viessem a comprometer todo o trabalho realizado até aquele momento.

A Figura 1 mostra um exemplo de imagem obtida pelo processo citado e que contém as informações referentes a um dos resultados da pesquisa. Como se pode notar, a identificação numérica de cada resultado foi circulada em azul, indicando publicações cujo tema se relaciona com o Ensino de Astronomia e, em vermelho, aquelas em que essa relação não foi observada, isto é, que se referem a publicações relacionadas a outras ciências catalogadas como ensino ou educação, ou à Astronomia pura e aplicada. Nesses casos, as publicações foram descartadas dos passos posteriores de análise. 


\section{Figura 1 - Exemplo de dado coletado no portal da CAPES}

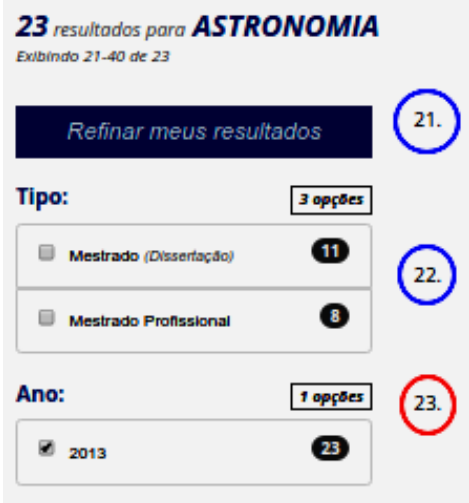

OTOFUJI, MAYSE. A experiência museal do Museu Dinâmico Interdisciplinar (MUDI/UEM): análise de visitas escolares 2012' 27/02/2013 255 f. Mestrado em EDUCACCAO PARA A CIENNCIA EA MATEMÁTICA Instituiç̧̆o de Ensino: UNIVERSIDADE ESTADUAL DE MARINGA, Maringá Biblioteca Depositária: Biblioteca Central da UNIVERSIDADE ESTADUAL DE MARINGÁ Detalhes

MORAIS, SILVILENE DE BARROS RIBEIRO. Museu de Ciência: o diálogo com as diferenças' 24/09/2013 231 f. Mestrado em EDUCAÇAO Instituiçăo de Ensino: UNIVERSIDADE FEDERAL DO RIO DE JANEIRO, Rio de Janeiro Biblioteca Depositária: Biblioteca do CFCH Detalhes

SANTA, FERNANDO DALA. justiça, política e formação na República platônica: a Paidéia enquanto caminho para a virtude' 30/OB/2013 110 f. Mestrado em EDUCAÇAO Instituição de Ensino: Fundação Universidade de Passo Fundo, Passo Fundo Biblioteca Depositária: Biblioteca Central Detalhes

Todas as informações referentes aos dados obtidos foram organizadas em uma planilha eletrônica semelhante ao MSExcel da Microsof TM, recomendada por Meyer e Avery (2009) e Felice e Janesick (2016) como uma ferramenta de análise de texto, além de manipulações numéricas. Novamente optou-se por um software livre, o WPS Spreadsheets que, segundo a Kingsoft Corporation (2016), consiste em uma planilha eletrônica, similar ao MSExcel e que atendeu perfeitamente aos objetivos da pesquisa. Ressalta-se que o uso de programas simplificou consideravelmente o processo de exploração dos dados, procedimento este que, segundo Bardin (2016), embora simples, constitui uma fase longa e fastidiosa quando realizada manualmente. Destaca-se também o papel destes programas na redução do consumo de papel e do tempo gasto na análise dos dados.

Finalizada a etapa de catalogação dos resultados obtidos, iniciou-se a sua classificação como Tese de Doutorado (D) ou Dissertação de Mestrado (M) e, paralelamente, a determinação do ano correspondente à publicação e da instituição de origem de cada um.

O passo seguinte foi caracterizado pela criação de um cadastro contendo o resumo de cada Tese e Dissertação, visando possibilitar a sua consulta sem a necessidade de conexão com a internet. Além disso, também foram inseridos nesse cadastro o nome do orientador, as palavras-chave, a área de concentração, a linha de pesquisa e o programa de pesquisa ao qual cada publicação se achava vinculada.

Terminada a elaboração do cadastro dos resumos, iniciou-se a leitura atenta dos mesmos, buscando-se extrair deles as informações que constituiriam os dados de análise do presente trabalho e que apresentadas ao longo deste artigo. 


\section{APRESENTAÇÃO E ANÁLISE DOS RESULTADOS}

Adota-se nesta seção, uma organização similar àquela adotada por autores que serviram de referência para este trabalho, dentre eles, Bretones e Megid Neto (2005).

\subsection{Resultados por ano}

O levantamento das Teses e Dissertações resultou na identificação de 289 publicações, das quais, 27 (9,3\%) são Teses de Doutorado e 262 (90,7\%) são Dissertações de Mestrado. A Tabela 1 apresenta a distribuição dos resultados obtidos, separados por tipo e em função do ano de publicação.

Tabela 1 - Teses e Dissertações por ano de publicação

\begin{tabular}{lllllllll}
\hline ANO & 2013 & 2014 & 2015 & 2016 & 2017 & 2018 & 2019 & TOTAL \\
\hline TESES & 3 & 3 & 1 & 6 & 9 & 4 & 1 & 27 \\
\hline DISSERTAÇÕES & 21 & 23 & 40 & 56 & 55 & 56 & 11 & 262 \\
\hline \multicolumn{7}{c}{ Fonte: Dados da pesquisa. }
\end{tabular}

As informações apresentadas na Tabela 1 evidenciam o crescimento considerável do número total de publicações no período compreendido entre janeiro de 2013 e novembro de 2019, atingindo o seu máximo no ano de 2017, com o registro de 64 publicações, o que corresponde a $22,1 \%$ do total obtido. De forma semelhante, nesse mesmo ano ocorreu o máximo de Teses publicadas, totalizando nove delas, o que representa 33,3\% do total obtido para todo o período analisado. Esse crescimento parece estar associado ao aumento dos programas de pós-graduação existentes no Brasil, conforme evidenciado por FAPEG (2017). Essa tendência também é evidenciada quando se analisam os totais de publicações por ano, representados graficamente.

A Figura 2 representa o número total de resultados obtidos em função do ano de sua publicação. Nessa mesma figura encontra-se uma linha tracejada que representa a tendência de crescimento do número de publicações com o passar do tempo. Essa linha foi obtida através do ajuste dos pontos, por meio da análise de regressão linear, descrita por Gupta (2002) e Lapponi (2004), utilizando-se planilhas eletrônicas. 


\section{Figura 2 - Total de resultados por ano de publicação}

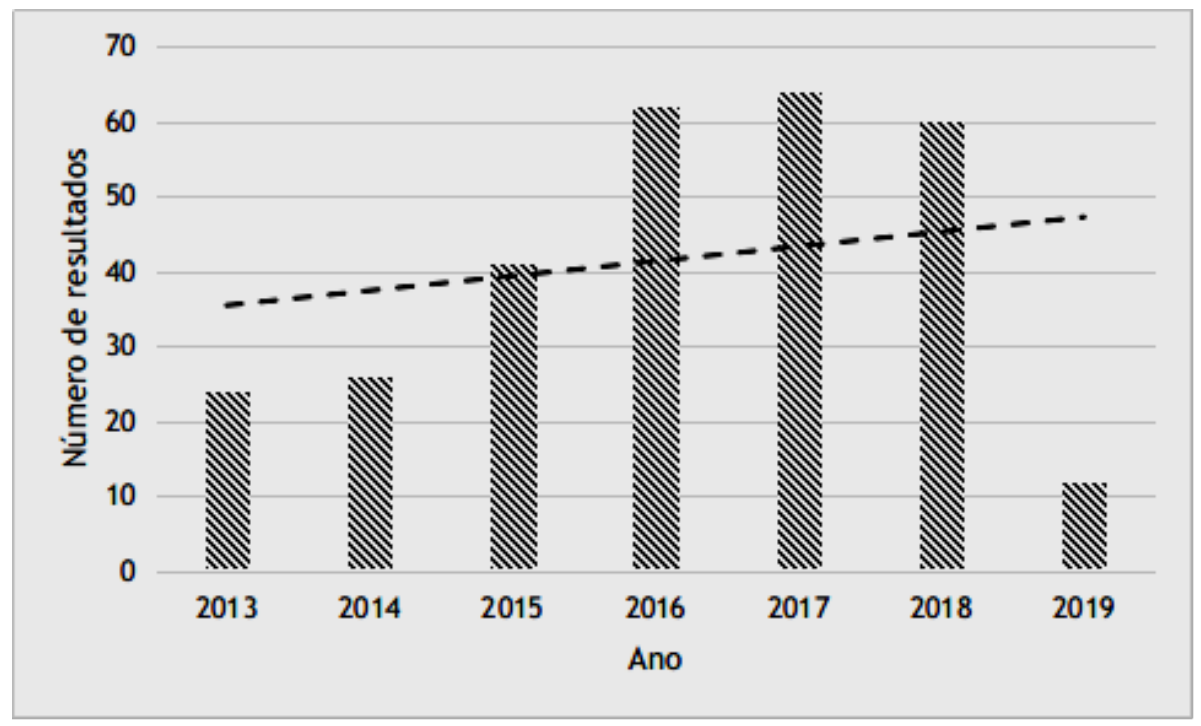

Fonte: Dados da pesquisa.

A observação atenta do gráfico evidencia um crescimento expressivo no total de publicações entre os anos de 2013 e 2016. Destaca-se o aumento do número de publicações, observado nos anos de 2015 e 2016, que pode estar relacionado à avaliação quadrienal realizada pela CAPES. É possível que esse crescimento também tenha relação com o aumento do total de bolsistas financiados pela CAPES e pelo Conselho Nacional de Desenvolvimento Científico e Tecnológico (CNPq) no período de 2004 a 2015 (JARDIM, 2020). A partir de 2016, observa-se que o total de publicações se manteve praticamente constante. Essa tendência pode estar relacionada à redução do número de bolsistas a partir de 2015, conforme ressalta Jardim (2020).

Destaca-se, ainda, o baixo número de publicações correspondentes ao ano de 2019. No entanto, ressalta-se que esse resultado pouco expressivo, com apenas 12 publicações $(4,2 \%)$, possa estar relacionado à falta de atualização dos Bancos de Teses e Dissertações que foram utilizados no levantamento dos dados e que não havia ocorrido até a data do término do presente trabalho, em novembro de 2019.

\subsection{Instituições de origem}

Quanto à Instituição de Ensino de origem, identifica-se que as Teses e Dissertações catalogadas são provenientes de 71 instituições, localizadas ao longo de todo o território nacional. A Tabela 2 contém a distribuição desses resultados em relação às Instituições de origem.

Como se pode notar, a Universidade de São Paulo (USP), com 43 publicações (14,9\%), é a instituição com o maior número de resultados obtidos no período considerado no levantamento, seguida pela Universidade Estadual de Feira de Santana (UEFS), com 41 publicações $(14,2 \%)$. 
Tabela 2 - Distribuição dos resultados por Instituição de Ensino

\begin{tabular}{l|l|l|l|l|l}
\hline INSTITUIÇÃO & N & INSTITUIÇÃO & N & INSTITUIÇÃO & N \\
\hline USP & 43 & UFSC & 3 & UEPB & 1 \\
\hline UEFS & 41 & EEL & 2 & UERJ & 1 \\
\hline UNESP & 20 & FUVATES & 2 & UERN & 1 \\
\hline UFES & 11 & IFCE & 2 & UERR & 1 \\
\hline UFRN & 10 & UEG & 2 & UESC & 1 \\
\hline UFSCAR & 10 & UEL & 2 & UFAM & 1 \\
\hline UNICSUL & 8 & UEMS & 2 & UFERSA & 1 \\
\hline UFRGS & 8 & UFBA & 2 & UFLA & 1 \\
\hline UFF & 7 & UFC & 2 & UFMT & 1 \\
\hline UFABC & 6 & UFJF & 2 & UFOP & 1 \\
\hline UNB & 6 & UFMG & 2 & UFPE & 1 \\
\hline UTFPR & 6 & UFPA & 2 & UFPI & 1 \\
\hline UEM & 5 & UFRPE & 2 & UFPR & 1 \\
\hline UFU & 5 & UFSM & 2 & UFRRJ & 1 \\
\hline UNICAMP & 5 & UNIFRA & 2 & UFT & 1 \\
\hline UNIFEI & 5 & UNIOESTE & 2 & UNIBAN & 1 \\
\hline UNIPAMPA & 5 & CUML & 1 & UNICENTRO & 1 \\
\hline UFG & 4 & IFES & 1 & UNIFAL & 1 \\
\hline UFMS & 4 & IFG & 1 & UNILA & 1 \\
\hline UFS & 4 & IFSUL & 1 & UNIMEP & 1 \\
\hline PUC-MG & 3 & PUC-RS & 1 & UNINTER & 1 \\
\hline UECE & 3 & UCS & 1 & UNIRIO & 1 \\
\hline UFAL & 3 & UDESC & 1 & UNIVASF & 1 \\
\hline UFRJ & 3 & UEA & 1 & TOTAL & $\mathbf{2 8 9}$ \\
\hline & & Fonte: Dados da pesquisa. &
\end{tabular}

Destacam-se, também, a Universidade do Estado de São Paulo (UNESP), com 20 publicações (6,9\%), a Universidade Federal do Espírito Santo (UFES), com 11 publicações (3,8\%), a Universidade Federal do Rio Grande do Norte (UFRN), a Universidade Federal de São Carlos (UFSCar), ambas com 10 publicações (3,5\%), a Universidade Cruzeiro do Sul (UNICSUL) e a Universidade Federal do Rio Grande do Sul (UFRGS), com 8 publicações $(2,8 \%)$ cada. No outro extremo, das 71 Instituições de Ensino relacionadas, 31 delas (43,7\%) apresentam apenas uma publicação e outras $15(21,1 \%)$ apresentam duas publicações cada. Esse resultado evidencia uma distribuição não homogênea das Teses e Dissertações em relação às Instituições de Ensino.

\subsection{Resultados por região}

Tomando como referência as Instituições de Ensino, foi possível identificar as regiões de origem das publicações. A Figura 3 apresenta o panorama da distribuição dos resultados obtidos ao longo das regiões do Brasil. 
Figura 3 - Teses e Dissertações por região

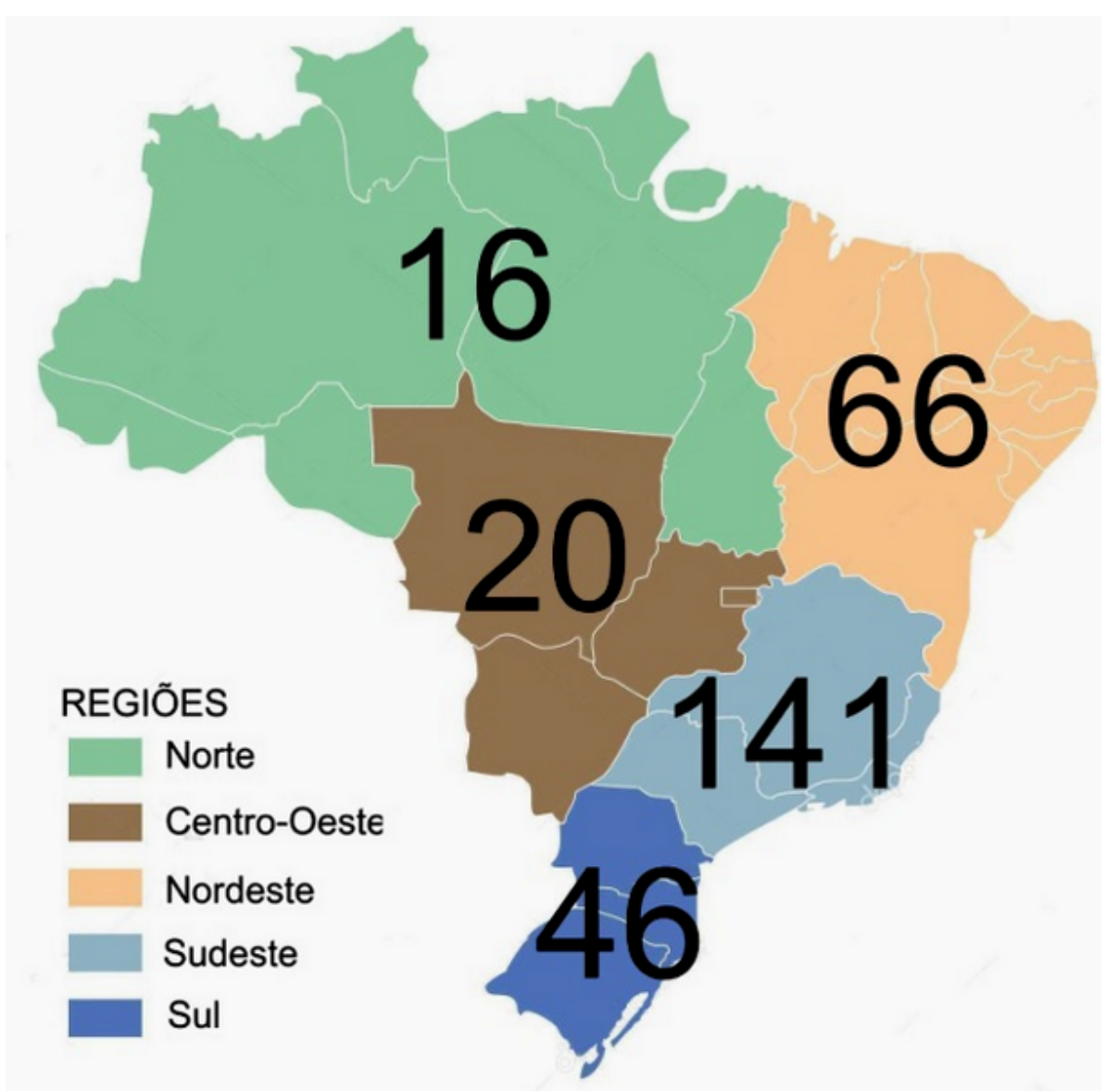

Fonte: Adaptado de Depositphotos (2018).

A Figura 3 ressalta a grande quantidade de publicações originárias da região sudeste do Brasil, com 141 publicações $(48,8 \%)$. Esse fato pode ter sua origem na grande concentração de Instituições de Ensino, chegando a 26 delas, o que corresponde a 36,6\% de todas as Instituições identificadas nesta pesquisa. A região nordeste vem em seguida, com 66 resultados, dos quais, $41(62,1 \%)$ são provenientes da UEFS, localizada no Estado da Bahia. As regiões norte e centro-oeste apresentam os menores totais de resultados catalogados neste trabalho.

\subsection{Resultados por Nível de Ensino}

Os resultados foram classificados quanto ao nível escolar abrangido. A referência para essa classificação encontra-se em Megid Neto (1999), Bretones e Megid Neto (2005) e em Bussi e Bretones (2013). Assim como fizeram Bussi e Bretones (2013), necessitou-se da adequação das categorias originais para possibilitar a classificação dos resultados, inclusive com a criação de novas categorias, no caso, "Ensino Técnico", "Não se aplica" e "Não identificado". Na categoria "Não se aplica" foram classificados os resultados que não estão relacionados a nenhum nível de ensino, como é o caso de alguns Estados da Arte e de algumas pesquisas histórico/bibliográficas. Em "Não identificado" encontram-se os resultados cujos resumos não evidenciaram qualquer informação que possibilitasse a identificação do nível de ensino abrangido. Na catego- 
ria "Geral" foram incluídos os resultados que abrangem a formação continuada de professores, educação especial, educação de jovens e adultos (EJA), educação não formal direcionada ao público geral etc.

A Figura 4 representa o resultado da classificação das publicações em relação ao nível de ensino, identificados pela seguinte simbologia EM (Ensino Médio), G (Geral), EF-2 (Ensino Fundamental 2), EF-1 (Ensino Fundamental 1), ES (Ensino Superior), ET (Ensino Técnico), EI (Ensino Infantil), NA (Não se aplica) e NI (Não identificado).

Figura 4 - Teses e Dissertações por região

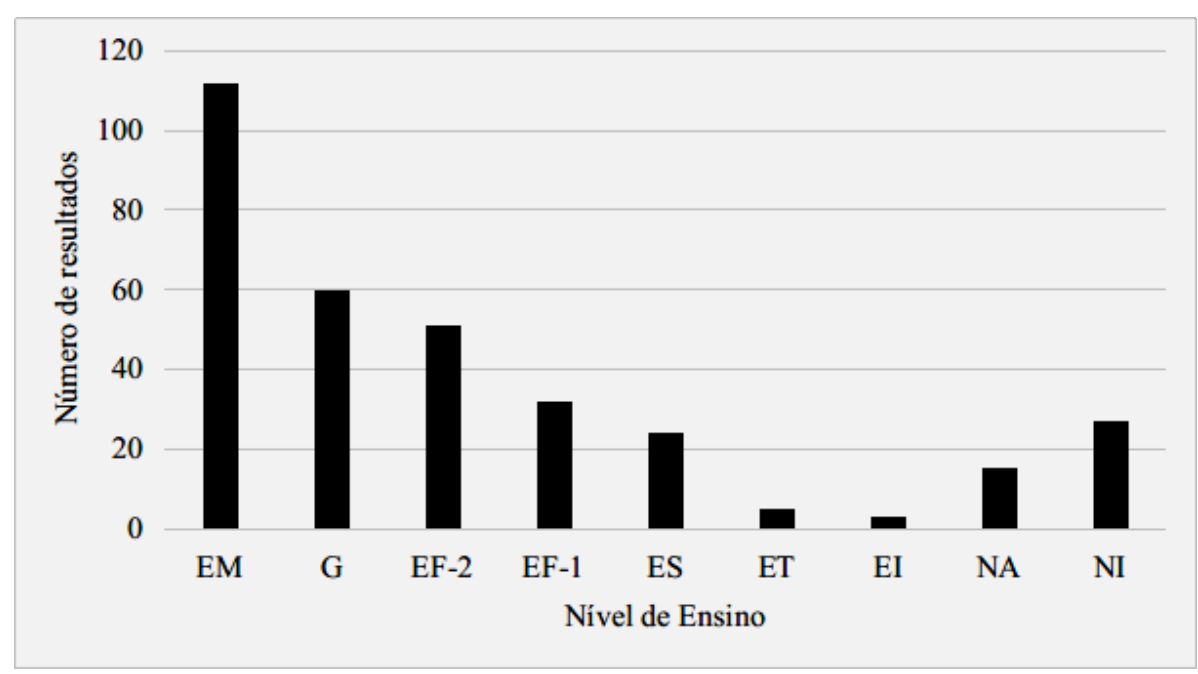

Fonte: Dados da pesquisa.

A Figura 4 evidencia a predominância de trabalhos voltados para o Ensino Médio em que foram classificadas $112(38,8 \%)$ das 289 publicações. Observa-se também, que o Ensino Técnico, com apenas cinco publicações $(1,7 \%)$ e o Ensino Infantil, com três publicações $(1,0 \%)$ carecem de estudos sobre o Ensino de Astronomia, o que indica um universo de pesquisa ainda pouco explorado. Ressalta-se ainda o percentual considerável de publicações em que o nível de ensino não pode ser determinado. Isso ocorreu porque os seus resumos, objetos de estudo desta pesquisa, não continham informações suficientes que permitissem essa identificação. Trata-se, portanto, de um indicativo da necessidade de um maior rigor na sua elaboração.

O resultado apresentado está de acordo com aqueles obtidos por Bretones e Megid Neto (2005) e por Bussi e Bretones (2013), exceto pela quantidade de publicações relacionadas ao Ensino de Astronomia voltadas para o Ensino Médio, que passaram a superar, em muito, aquelas relacionadas aos demais níveis de ensino. Esse fato chamou a atenção e parece indicar que, a partir de 2013, houve um crescimento acentuado de Teses e Dissertações voltadas para o Ensino Médio. Com o objetivo de verificar essa possível tendência, foi realizado um estudo estatístico específico para esse nível de ensino, cujo resultado encontra-se representado na Figura 5. 


\section{Figura 5 - Tendência das Teses e Dissertações voltadas para o Ensino Médio}

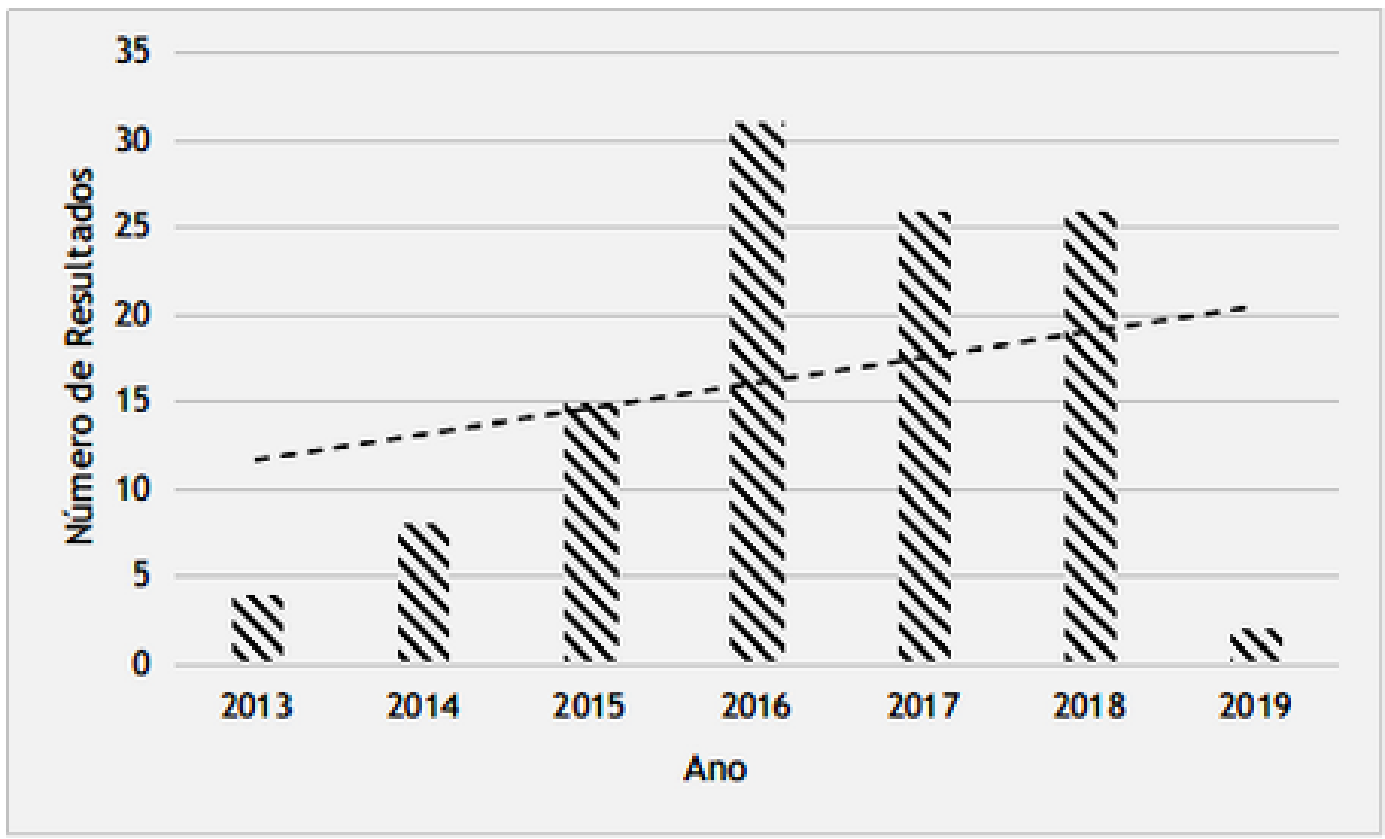

Fonte: Dados da pesquisa.

De fato, observando-se a linha de tendência (linha tracejada), obtida através do procedimento já descrito, confirma-se o crescimento do total de Teses e Dissertações direcionadas ao Ensino Médio no período analisado. Vale a pena ressaltar que Bretones e Megid Neto (2005) observaram uma frequência muito baixa de publicações voltadas para o Ensino Médio. No entanto, alguns anos mais tarde, Bussi e Bretones (2013) apresentaram em seu artigo um novo panorama para o Ensino Médio, caracterizado pelo crescimento considerável do número de publicações voltadas para esse nível de ensino. Os resultados aqui apresentados estão de acordo com observações de Bussi e Bretones (2013) e parecem corroborá-las.

\subsection{Resultados por Programas de Pesquisa}

Tomando-se como foco de análise os programas de pesquisa aos quais as Teses e Dissertações se acham vinculados, foram identificados 39 diferentes programas, cujas denominações foram obtidas diretamente dos Bancos de Teses e Dissertações analisados, sobretudo o Catálogo de Teses e Dissertações da CAPES. Optou-se por manter as denominações dadas a esses programas em seus formatos originais, isto é, com o mesmo formato com que foram catalogados pelos seus respectivos autores nos Bancos analisados. Ressalta-se, entretanto, a existência de nomes distintos que parecem designar um mesmo programa de pesquisa. É o caso, por exemplo, dos programas Educação em Ciências e Matemática e Educação para a Ciência e Matemática. Ainda assim, essas designações foram mantidas. Na Tabela 3 encontram-se relacionados esses 39 programas, identificados por um sistema de siglas e por sua denominação original. 
Tabela 3 - Distribuição dos resultados por Instituição de Ensino

\begin{tabular}{l|l|l|l|l|l}
\hline & SIGLA & PROGRAMA DE PESQUISA & & SIGLA & PROGRAMA DE PESQUISA \\
\hline 1 & ENF & Ensino de física & 21 & ENHCM & Ensino e história das ciências e da matemática \\
\hline 2 & AST & Astronomia & 22 & EDCEM & Educação científica e matemática \\
\hline 3 & ENC & Ensino de ciências & 23 & PEC & Projetos educacionais em ciências \\
\hline 4 & ENAST & Ensino de astronomia & 24 & DCC & Divulgação científica e cultural \\
\hline 5 & ED & Educação & 25 & EDT & Educação e tecnologia \\
\hline 6 & ENCM & Ensino de ciências e matemática & 26 & EDCT & Educação científica e tecnológica \\
\hline 7 & EDPC & Educação para a ciência & 27 & ENCN & Ensino de ciências naturais \\
\hline 8 & ENCNM & Ensino de ciências naturais e matemática & 28 & EDM & Educação matemática \\
\hline 9 & EN & Ensino & 29 & SCF & Sociedade, cultura e fronteiras \\
\hline 10 & EDC & Educação em ciências & 30 & ICAL & Integração contemporânea e América latina - ICAL \\
\hline 11 & MRN & Matemática em rede nacional & 31 & CA & Computação aplicada \\
\hline 12 & EDCM & Educação em ciências e matemática & 32 & MP & Museologia e patrimônio \\
\hline 13 & EDPCM & Educação para a ciência e matemática & 33 & DECM & Docência em educação em ciências e matemática \\
\hline 14 & ENCE & Ensino de ciências exatas & 34 & EFHC & Ensino, filosofia e história das ciências \\
\hline 15 & FCET & Formação científica educacional e tecnológica & 35 & EDNT & Educação e novas tecnologias \\
\hline 16 & ENCT & Ensino de ciências e tecnologia & 36 & ENCHSN & Ensino de ciências humanas, sociais e da natureza \\
\hline 17 & GEO & Geografia & 37 & ENEB & Ensino na educação básica \\
\hline 18 & ENCDN & Ensino de ciências da natureza & 38 & ENCH & Ensino de ciências e humanidades \\
\hline 19 & EDMEF & Educação matemática e ensino de física & 39 & NE & Não especificado \\
\hline 20 & ENCEM & Ensino de ciências e educação matemática & & & \\
\hline
\end{tabular}

Fonte: Dados da pesquisa.

O sistema de siglas apresentado na Tabela 3 foi elaborado pelos autores da presente pesquisa, tendo como referência as iniciais das palavras que formam os nomes dos programas de pesquisa, tomando-se o cuidado de diferenciar da forma mais lógica possível aqueles cujos nomes se assemelhassem demasiadamente. Esse sistema de siglas possibilitou a elaboração de uma representação gráfica para a distribuição das Teses e Dissertações em função de seus respectivos programas de pesquisa. Essa representação encontra-se na Figura 6.

Figura 6 - Resultados por Programa de Pesquisa

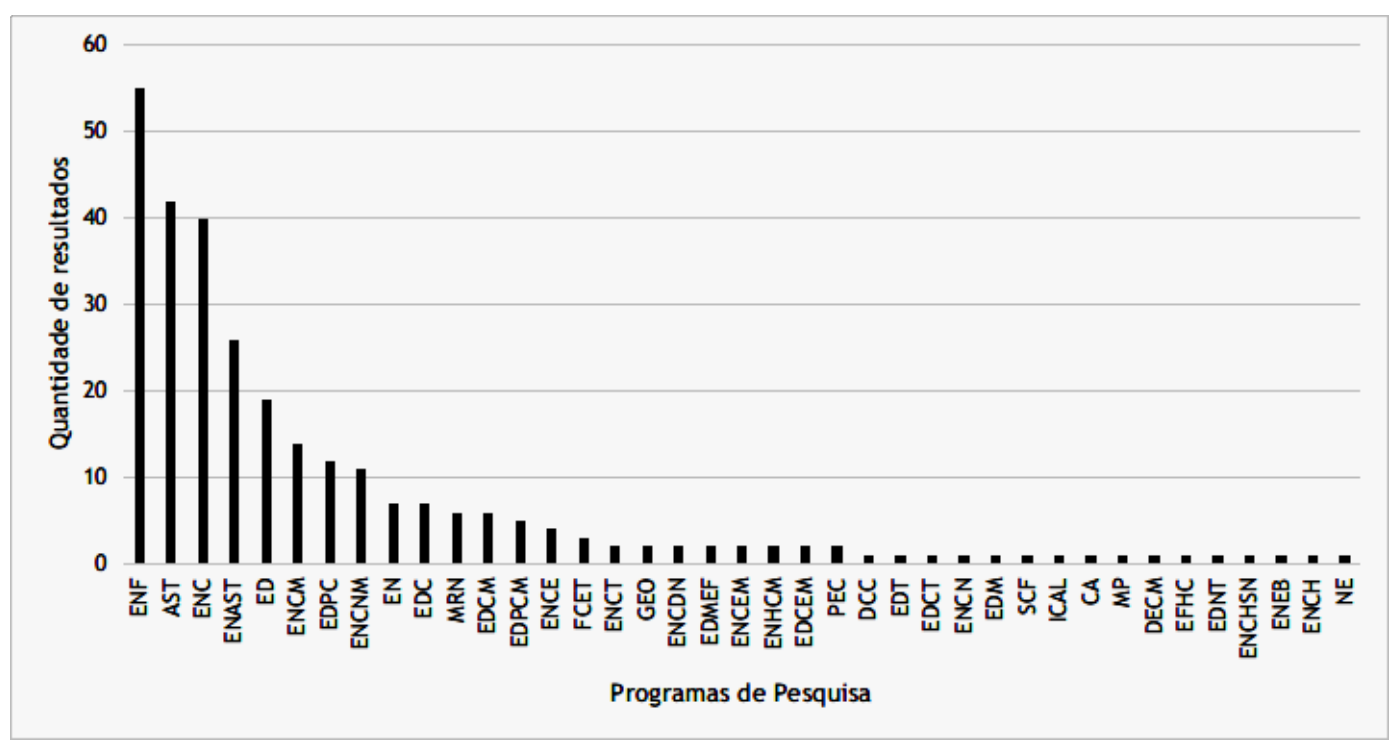

Fonte: Dados da pesquisa.

A análise da Figura 6 permite concluir que grande parte das Teses e Dissertações identificadas no levantamento realizado encontram-se cadastradas nos programas de Ensino de Fí- 
sica (ENF), com um total de 55 publicações (19,0\%), Astronomia (AST) com 42 publicações (14,5\%), Ensino de Ciências (ENC) com 40 publicações (13,8\%) e Ensino de Astronomia (ENAST) com 26 publicações $(9,0 \%)$. Esses programas contabilizam juntos, mais de 56,4\% de todos os resultados obtidos, o que evidencia a preocupação dos pesquisadores com o Ensino de Ciências e, em especial, com o Ensino de Astronomia.

\subsection{Resultados por foco temático}

Ainda com referência nos levantamentos feitos por Bretones e Megid Neto(2005) e por Bussi e Bretones (2013), realizou-se a classificação dos resultados de acordo com o seu foco temático. Para tanto, foram empregados os mesmos critérios de classificação utilizados por Megid Neto (1999) e por Bretones e Megid Neto (2005). Houve, entretanto, durante o procedimento de análise, a necessidade de criação de mais dois focos temáticos, que permitiram a classificação de alguns resultados que não se mostraram perfeitamente compatíveis com aqueles já existentes. Esses novos focos temáticos são "Divulgação Científica" e "Estado da Arte ou do Conhecimento".

Realizou-se a leitura criteriosa de Megid Neto (1999) e de Soares e Maciel (2000) com o objetivo de estabelecer a correta compreensão dos significados atribuídos a cada um dos focos temáticos empregados na classificação dos dados. No entanto, é importante ressaltar que o processo de classificação não está isento das influências exercidas pelos estados gerais do pesquisador e, ainda que de forma imperceptível, de alguns vieses como o de busca e o de seleção (ZOLTOWSKI et al., 2014) que podem, de certa forma, manifestar-se na pesquisa ou na interpretação dos dados. Assim sendo, certamente poderão ser observadas algumas pequenas divergências entre o panorama expresso neste trabalho e aqueles evidenciados em trabalhos já citados.

Na Tabela 4 encontram-se relacionados os focos temáticos e o total de resultados nele classificados.

\section{Tabela 4 - Distribuição dos resultados por Instituição de Ensino}

\begin{tabular}{l|l|l}
\hline FOCOS TEMÁTICOS & N & $\%$ \\
\hline CONTEÚDO-MÉTODO & 235 & 81,3 \\
\hline FORMAÇÃO DE CONCEITOS & 234 & 81,0 \\
\hline RECURSOS DIDÁTICOS & 233 & 80,6 \\
\hline CURRIÍCULO E PROGRAMAS & 50 & 17,3 \\
\hline FORMAÇÃO DE PROFESSORES/INSTRUTORES & 47 & 16,3 \\
\hline ENSINO NÁO-FORMAL (NÃO ESCOLAR) & 46 & 15,9 \\
\hline CARACTERÍSTICAS E CONCEPÇÕES DO ALUNO & 44 & 15,2 \\
\hline CARACTERÍSTICAS E CONCEPÇÕES DO PROFESSOR & 28 & 9,7 \\
\hline DIVULGAÇÃO CIENTÍFICA & 26 & 9,0 \\
\hline ESTADO DA ARTE OU DO CONHECIMENTO & 14 & 4,8 \\
\hline
\end{tabular}

Fonte: Dados da pesquisa.

De certa forma os dados aqui apresentados estão em concordância com aqueles apresentados por Bretones e Megid Neto (2005) e por Bussi e Bretones (2013). No entanto, como já mencionado, pequenas divergências poderão ser observadas, sobretudo quanto ao montante 
de trabalhos classificados no foco temático Formação de Conceitos. Essa divergência pode ter como possível explicação, os vieses epistemológicos dos pesquisadores envolvidos.

Observando-se a Tabela 4, é possível notar que grande parte das publicações analisadas foram classificadas nos três focos temáticos, a saber, Conteúdo-Método, Formação de Conceitos e Recursos didáticos. Como exemplos de trabalhos classificados nesses três focos temáticos temos Muller (2013), Macêdo (2014), Boaventura (2015), Maciel (2016) e Vassoler (2017). A grande frequência de publicações nesses três focos evidenciam a tendência dos autores em direcionarem sua pesquisa para elaboração de conteúdos, para o desenvolvimento de novas metodologias e para a produção de recursos didáticos, tendo como principal objetivo a formação de conceitos fundamentais em Astronomia. Além disso, há uma quantidade considerável de resultados que têm como focos temáticos a Formação de Professores/Instrutores, o Ensino Não-Formal e as Características e Concepções de Alunos, o que reforça a preocupação dos pesquisadores com o Ensino de Astronomia. Dentre os trabalhos classificados em um desses focos temáticos ou em mais de um deles temos, como exemplos, Diniz (2013), Barros (2014), Fontanella (2015) e Silva (2018).

Os resultados apresentados nesta seção também estão de acordo com Bretones e Megid Neto (2005).

\section{CONSIDERAÇÕES FINAIS}

Espera-se que o presente trabalho de pesquisa tenha possibilitado ao leitor vislumbrar o panorama das produções acadêmicas voltadas para o Ensino de Astronomia no Brasil, no período de janeiro de 2013 a novembro de 2019. Destacam-se, a seguir, algumas das considerações que emergiram do processo de análise das Teses e Dissertações:

- O número de produções científicas relacionadas ao Ensino de Astronomia continua a aumentar, o que evidencia um crescente interesse pelo tema por parte da comunidade científica.

- Identificação de publicações acadêmicas voltadas para o Ensino de Astronomia em todo o território nacional, com destaque para a região sudeste do Brasil.

- Crescimento considerável das publicações sobre Ensino de Astronomia voltadas para o nível Médio da Educação Básica, além do aumento dessas publicações nos demais níveis de ensino.

- Distribuição das publicações em diversos programas de pesquisa, destacando-se os programas que abordam o Ensino de Física, o Ensino de Ciências e o Ensino de Astronomia.

- Grande frequência de publicações que têm o seu foco temático direcionado para a 
elaboração de conteúdos e para o desenvolvimento e a produção de recursos didáticos, tendo como principal objetivo, a formação de conceitos.

Durante a leitura dos resumos, foi possível constatar que, em grande parte deles, há uma carência de informações essenciais, o que dificultou a classificação de algumas publicações analisadas, chegando a inviabilizá-la, em alguns casos. Trata-se, portanto, de um indicador da necessidade de uma elaboração mais rigorosa dos resumos, a fim de que forneçam um bom panorama da pesquisa apresentada.

Por fim, ressalta-se que estudos do tipo Estado da Arte são de grande importância para o Ensino de Astronomia, uma vez que evidenciam os aspectos da área da educação e os temas que mais preocupam os pesquisadores (ROMANOWSKI; ENS, 2006). Esse tipo de estudo, segundo Romanowski e Ens (2006), pode apresentar experiências inovadoras, que propõem alternativas para solucionar os problemas relacionados à prática, além de apontarem novas metodologias e recursos didáticos que o professor pode agregar à sua prática pedagógica com o objetivo de tornar o processo ensino-aprendizagem mais dinâmico e mais eficaz. Entretanto, é necessário que os resultados desses estudos cheguem até o ambiente escolar, mais precisamente, à sala de aula, local onde as estratégias de Ensino se convertem, de fato, em realidade. 


\section{REFERÊNCIAS}

AFONSO, G. B.; NADAL, C. A. Arqueoastronomia no Brasil. In: Matsuura, O. T. (org.). História da astronomia no Brasil. Recife: Companhia de Pernambuco - Cepe, 2013. p. 52-86., n. 3.

BARBOSA, J. I. L.; VOELZKE, M. R. Questionário-diagnóstico sobre conceitos básicos de astronomia por alunos do ensino médio integrado. Revista de Ensino de Ciências e Matemática, v. 7, n. 2, p. 25-38, 2016.

BARDIN, L. Análise de conteúdo. Lisboa: Editora Edições 70, 2016.

BARROS, M. F. Os movimentos dos planetas e os modelos de Universo: uma proposta de sequência didática para o Ensino Médio. 2014. Dissertação (Mestrado Profissional) - Universidade Federal do Espírito Santo, Vitória, 2014.

BATISTA, M. C. Um estudo sobre o Ensino de Astronomia na formação inicial de professores dos anos iniciais. Cadernos da Pedagogia, v. 9, n. 18, 2016.

BOAVENTURA, G. A. S. O uso do dispositivo de Orrery no Ensino de Astronomia no Ensino Médio. 2015. Dissertação (Mestrado) - Universidade Federal Fluminense, São Paulo, 2015.

BRASIL. Ministério da Ciência e Tecnologia. Comissão Especial de Astronomia. Plano Nacional de Astronomia. Brasília: 2010.

BRASIL. Ministério da Educação. Secretaria de Educação Básica. Base Nacional Comum Curricular. Brasília, DF, 2018.

BRETONES, P. S. Disciplinas introdutórias de Astronomia nos cursos superiores do Brasil. 1999. Dissertação (Mestrado) - Instituto de Geociências, Universidade de Campinas, Campinas, 1999.

BRETONES, P. S.; MEGID NETO, J. Tendências de teses e dissertações sobre Educação em Astronomia no Brasil. Boletim da Sociedade Astronômica Brasileira, v. 24, n. 2, p. 35-43, 2005.

BUSSI, B.; BRETONES, P. S. Educação em Astronomia nos trabalhos dos ENPECs de 1997 a 2011. In: ENCONTRO NACIONAL DE PESQUISA EM EDUCAÇÃO EM CIÊNCIAS, 9., p. 1-8, 2013, Aguas de Lindóia. Anais[...]. Rio de Janeiro, 2013,.

COELHO, F. B. O; BULEGON, A. M. Análise do tema astronomia, nos livros didáticos indicados pelo PNLD, dos anos iniciais do Ensino Fundamental. VIDYA, v. 33, n. 1, p. 12, 2013.

DANG, T.; DANG, C.; WATTS, L. Manual do KolourPaint. 2011. Tradução Marcus Gama e André Marcelo Alvarenga. Disponível em: <https://docs.kde.org/stable5/pt_BR/ kdegraphics/kolourpaint/index.html>. Acesso em: 10 out. 2018.

DARROZ, L. M.; BAGESTAN, G. H.; DA ROSA, C. T. W. Conceitos de astronomia presentes em livros didáticos de física. Caderno de Física da UEFS, v.15, n. 1, p. 1501.1-12, 2017. 
DEPOSITPHOTOS. Mapa do Brasil com as divisões dos Estados e Regiões. 2018. Disponível em: $<$ https://br.depositphotos.com/230939992/stock-illustration-map-brazildivisions-states. html>. Acesso em: 25 out. 2019.

DINIZ, A. C. S. Ensino de Ciências em ambientes não escolares: desenvolvimento do modelo de visitas educativas na exposição de Astronomia do Museu de Ciências Naturais da PUC Minas. 2013. Dissertação (Mestrado profissional) - Pontifícia Universidade Católica de Minas Gerais, Belo Horizonte, 2013.

FAPEG. Pós-graduação brasileira teve avanço qualitativo na última década. 2017. Disponível em: <http://www.fapeg.go.gov.br/pos-graduacao-brasileira-teve-avanco-qualitativo-naultima-decada/>. Acesso em: 16 jan. 2021.

FELICE, D.; JANESICK, V. J. Using spreadsheets as a qualitative analysis tool. 2016. Disponível em: <https://digitaltoolsforqualitativeresearch.org/2016/11/28/usingspreadsheets-as-a-qualitative-analysis-tool/>. Acesso em: 25 out. 2018.

FERREIRA, N. S. A. As pesquisas denominadas “Estado da Arte". Educação \& sociedade, v. 23, p. 257-272, 2002.

FERREIRA, O. R.; VOELZKE, M. R. Análise do banco de dados de teses e dissertações do DME/UFSCAR sobre Educação em Astronomia. Revista Univap, v. 19, n. 34, p. 16-20, 2013.

FONTANELLA, D. Ensino de Astronomia: investigando a formação docente em um espaço não-formal. 2015. Dissertação (Mestrado) - Universidade Estadual do Oeste do Paraná, Cascavel, 2015.

FRAKNOI, A. The state of astronomy education in the US. In: Astronomy education: Current developments, future coordination. ed. J.R. Percy, ASP Conf. Series, v. 89, p. 9, 1996.

GAMA, L. D; HENRIQUE, A. B. Astronomia na sala de aula: por quê?. Revista Latino-Americana de Educação em Astronomia, n. 9, p. 7-15, 2010.

GEBARA, M. J. F. A formação continuada de professores de Ciências: contribuições de um curso de curta duração com tema geológico para uma prática de ensino interdisciplinar. 2009. Tese (Doutorado) - Universidade Estadual de Campinas, Instituto de Geociências, Campinas, 2009.

GONZAGA, E. P.; VOELZKE, M. R. Planetário digital móvel: Concepções astronômicas dos professores do litoral norte paulista. Educação \& Linguagem, v. 18, n. 1, p. 67-78. 2011.

GUPTA, V. Statistical analysis with Excel. Canada: VJ Books Inc., 2002.

HORVAT, J. E. O ABCD da Astronomia. São Paulo: Editora Livraria da Física, 2008.

IVANISSEVICH, A.; WUENSCHE, C. A.; ROCHA, J. F. V. Astronomia hoje. Rio de Janeiro: Instituto Ciência Hoje, 2010.

JARDIM, C. E. A expansão e o financiamento da pós-graduação no Brasil e a meta 14 do Plano Nacional de Educação. FINEDUCA - Revista de Financiamento da Educação, v. 10, 2020.

KINGSOFT, CORPORATION. (China) WPS Office: User Manual of WPS Office 2016. 2016. Disponível em: <https://help.wps.com/files/wps_spreadsheets_2016.pdf>. Acesso em: 04 dez. 2019. 
LANGHI, R. Astronomia nos anos iniciais do ensino fundamental: repensando a formação de professores. 2009. Tese (Doutorado) - Universidade Estadual Paulista, Faculdade de Ciências, Bauru, 2009.

LANGHI, R.; NARDI, R. Ensino de astronomia: Erros conceituais mais comuns presente em livros didáticos de ciência. Caderno Brasileiro de Ensino de Física, v. 24, n. 1, p. 87-111, 2007.

LANGHI, R.; NARDI, R. Justificativas para o ensino de astronomia: o que dizem os pesquisadores brasileiros?. Revista Brasileira de Pesquisa em Educação em Ciências, v. 14, n. 3, p. 041-059, 2014.

LAPPONI, J. C. Estatística usando Excel. Rio de Janeiro: Elsevier Brasil, 2005.

MACIEL, R. R. A Astronomia nas aulas de Física: uma proposta de utilização de Unidades de Ensino Potencialmente Significativas (UEPS). 2016. Dissertação (Mestrado profissional) - Universidade Federal de Santa Catarina, Araranguá, 2016.

MACÊDO, J. A. Formação inicial de professores de ciências da natureza e matemática e o ensino de astronomia. 2014. Tese (Doutorado em Ensino de Ciências e Matemática). Universidade Cruzeiro do Sul, São Paulo, 2014.

MACÊDO, J. A.; PEDROSO, L. S.; VOELZKE, M. R. Levantamento das abordagens e tendências dos trabalhos sobre as tecnologias de informação e comunicação apresentados no XIX Simpósio Nacional de Ensino de Física. Caderno Brasileiro de Ensino de Física, v. 31, n. 1, p. 167-197, 2014.

MACÊDO, J. A.; VOELZKE, M. R. As concepções prévias, os recursos tradicionais e as tecnologias digitais no ensino de astronomia. Imagens da Educação, v. 4, n. 3, p. 49-61, 2014.

MEGID NETO, J. Tendências da pesquisa acadêmica sobre o ensino de Ciências no nível fundamental. 1999. Tese (Doutorado) - Universidade Estadual de Campinas, Campinas, 1999.

MEYER, D. Z.; AVERY, L. M.; MEGID NETO, J. Excel as a qualitative data analysis tool. Field methods, v. 21, n. 1, p. 91-112, 2009.

MORAES A. C.; VOELZKE, M. R.; MACÊDO, J. A. Análise das concepções astronômicas apresentadas por alunos do Instituto Federal de São Paulo. - Campus Cubatão. Imagens da Educação, v. 6, n. 1, p. 99-106, 2016.

MORAIS, P. V.; MOREIRA, M. D.; SALES, N. L. L. Análise de erros conceituais e desatualizações de livros de ciências e geografia após a análise do PNLD. In: SIMPÓSIO NACIONAL DE EDUCAÇÃO EM ASTRONOMIA, 2., 2012, São Paulo. Atas [...]. São Paulo: USP, 2012.

MULLER, A. M. O Ensino de Fundamentos de Astronomia e Astrofísica na modalidade a distância para alunos de Graduação. 2013. Dissertação (Mestrado Profissional) - Universidade Federal do Rio Grande do Sul, Porto Alegre, 2013.

PEIXOTO, D. E. Astronomia como disciplina integradora para o Ensino de Ciências. Tese (Doutorado) - Universidade Estadual de Campinas, Instituto de Física Gleb Wataghin, Campinas, 2018. 
PEIXOTO, D. E.; RAMOS, E. M. F. Formação do professor de física para o ensino de astronomia: algumas possibilidades e reflexões. In: REUNIÃO ANUAL DA SOCIEDADE BRASILEIRA PARA O PROGRESSO DA CIÊNCIA-SBPC, 63., 2011, Goiânia. Anais[...]. Goiânia, 2011.

PERCY, J. R. Teaching astronomy? why and how? Journal of the American Association of Variable Star Observers (JAAVSO), v. 35, n. 1, p.248-254. 2006.

PEREIRA, F. K.; MACHADO, J. C. Iniciação à Informática Educativa. - Belém: EdUFPA, 2008.

PINTO, H. J. R.; HETEM, J. G; ALMEIDA, A. A.; BOMFIM, R.; GRUENWALD, V. J. P.; SARAIVA, M. D. F. O.; JAFELICE, L. C. Ensino de astronomia na graduação. Relatório Anual do INCT-A (anexo 5b), 2009. Disponível em: <http://www.astro.iag.usp.br/incta Rel_Anual_2009/Anexo_5b_Ensino-Astronomia.pdf>. Acesso em: 7 set. 2018.

PRIGOL, E. L. Pesquisa estado do conhecimento: Uma visão para a prática pedagógica e a formação de professores. In: CONGRESSO NACIONAL DE EDUCAÇÃO EDUCERE, 11., 2013, Curitiba. Anais[...]. Paraná: PUCPR, 2013.

ROMANOWSKI, J. P.; ENS, R. T. As pesquisas denominadas do tipo "Estado da Arte". Revista Diálogo Educacional, v. 6, n. 19, 2006.

SAVALL, A.; DUTRA, C. M. Astronomia no ensino superior: Qual sua evidência nos cursos de licenciatura em física e ciências da natureza? In: SALÃO INTERNACIONAL DE ENSINO, 6., PESQUISA E EXTENSÃO, 6., n. 1, 2014. Anais [...]. UNIPAMPA, 2014.

SIEMSEN, G. H.; LORENZETTI, L. A pesquisa em ensino de astronomia: analisando a produção acadêmica brasileira. In: ENCONTRO NACIONAL DE PESQUISA EM EDUCAÇÃO EM CIÊNCIAS, 11., 2017, Florianópolis. Atas[...]. Florianópolis: Universidade Federal de Santa Catarina, 2017.

SILVA, M. C. B. O Museu de Ciência como cenário da formação docente: saberes e concepções de licenciandos mediadores do Museu Seara da Ciência - UFC. 2018. Dissertação (Mestrado) - Universidade Federal do Ceará, Fortaleza, 2018.

SIMÕES, C. C. Elementos de Astronomia nos livros didáticos de Física. 2009. Dissertação (Mestrado Profissional) - Pontifícia Universidade Católica de Minas Gerais, Belo Horizonte, 2009.

SIMÕES, C. C.; VOELZKE, M. R. Aplicativos móveis e o ensino de astronomia. Research, Society and Development, v.9, n.10, p.e5089108920-e5089108920, 2020.

SOARES, M. B.; MACIEL, F. Importância e justificativa para o ensino de astronomia: Um olhar para as pesquisas da área. In: SIMPÓSIO DE EDUCAÇÃO EM ASTRONOMIA, 2., 2012, São Paulo. Atas [... ]. São Paulo, 2012.

SOARES, M. B.; MACIEL, F. Alfabetização. Brasília: MEC/Inep/Comped, 2000.

TEIXEIRA, C. H. S. Enfoque CTSA no ensino de astronomia: uma investigação de possibilidades por meio da astronáutica. 2013. Tese (Doutorado) - Universidade Estadual Paulista Julio de Mesquita Filho, Bauru; SP, 2013. 
VASSOLER, K. L. F. Ensino de Astronomia na Educação Integral: Reflexões e considerações sobre a formação de professores em Curitiba-PR. Gestão 2013-2016. 2017. Dissertação (Mestrado Profissional) - Centro Universitário Internacional, Curitiba, 2017.

ZICCARDI, L. R. N. O curso de matemática da Pontifícia Universidade Católica de São Paulo: uma história de sua construção/desenvolvimento/legitimação. 2009. Tese (Doutorado) - Pontifícia Universidade Católica de São Paulo, São Paulo, 2009.

ZOLTOWSKI, A. P. C.; COSTA, A. B. TEIXEIRA, M. A. P.; KOLLER, S. H. Qualidade metodológica das revisões sistemáticas em periódicos de psicologia brasileiros. Psicologia: Teoria e Pesquisa, v. 30, n. 1, p. 97-104, 2014. 